\title{
INFECÇÃO ALIMENTAR (VIA ORAL) POR TRYPANOSOMA CRUZI E SEUS IMPACTOS NA SAÚDE PÚBLICA: UMA REVISÃO
}

\author{
FOOD INFECTION (ORAL) BY TRYPANOSOMA CRUZI AND ITS \\ IMPACTS ON PUBLIC HEALTH: A REVIEW
}

\begin{abstract}
RESUMO: OBJETIVO: Realizar revisão de literatura com intuito de identificar a incidência da transmissão via oral da Doença de Chagas por meio de alimentos contaminados e seus impactos no âmbito da saúde publica. MÉTODO: Trata-se de uma revisão de literatura, que utilizou busca de acesso online às bases como Science Direct, Portal de Periódicos da Coordenação de Aperfeiçoamento de Pessoal de Nível Superior, Lilacs, Scielo, Medline, PubMed, Biblioteca Virtual em Saúde com os descritores "T. cruzi" "Epidemiologia" e/ou "Saúde Pública". RESULTADO: E possivel identificar que o numero de casos da doenca de chagas humana teve um crescimento significativo quando associado a transmissão por via oral, apresentado um cenario de risco quando analisado em alimentos como com a polpa do açai e caldo da cana de açucar. CONCLUSÃO: Com isso considera-se que se faz necessario a aplicabilidade de politicas publicas de saúde que possam contribuir para as ações de prevenção, promoção da saúde e controle da doença, alem da necessidade de disseminar o conhecimento das mais diversas formas de transmissão da doença e os seus riscos, tornando assim em evidencia a necessidade de uma abordagem multiprofissional, permitindo que a tematica permei por outras areas de atuação profissional de forma continua.
\end{abstract}

Palavras chave: Doença de Chagas; Triatomíneo; Vetor.

ABSTRACT: OBJECTIVE: To carry out a literature review in order to identify the incidence of oral transmission of Chagas Disease through contaminated food and its impact on public health. METHOD: This is a literature review that used online access to databases such as Science Direct, Portal of Journals of the Coordination for the

\footnotetext{
1 Discente da Pós-Graduação em Ambiente, Tecnologia e Sociedade; E-mail: enfermagem.ismael@gmail.com.

2 Docente da Universidade Federal Rural do Semi-Árido; E-mail: anacarla@ufersa.edu.br.
} 
Improvement of Higher Education Personnel, Lilacs, Scielo, Medline, PubMed, Virtual Health Library with descriptors "T. cruzi " Epidemiology "and / or" Public Health ". RESULT: It is possible to identify that the number of cases of human chagas disease has grown significantly when associated with oral transmission, presenting a risk scenario when analyzed in foods such as açai pulp and sugar cane juice. CONCLUSION: With this it is considered that it is necessary to apply public health policies that can contribute to prevention, health promotion and disease control actions, in addition to the need to disseminate knowledge of the most diverse forms of disease transmission and its risks, thus highlighting the need for a multiprofessional approach, allowing the thematic to permeate other areas of professional practice on a continuous basis.

Keywords: Chagas disease; Triatomine; Vector. 


\section{INTRODUÇÃO}

A Doença de Chagas é ocasionada pelo protozoário Trypanosoma cruzi e pode ser transmitida pelo vetor triatomíneo (McCLEAN et al., 2020), transmissão congênita (RIOS et al., 2020), transfusional, acidental e transplante (GÓMEZ et al., 2019) além da transmissão oral pela ingestão de alimentos contaminados (ZABALA et al., 2019).

Patologia endêmica em vários países da América (RASSI Jr. et al 2015) em razão do difícil diagnóstico, onde na fase aguda da infecção pode ocorrer falso negativo para testes sorológicos (sorologia por $\lg \mathrm{G}$ ), entretanto com diagnóstico positivo para microscopia parasitária (MAYTA et al., 2019). Na fase crônica, os protozoários raramente apresentam identificação no sangue com diagnóstico realizado, na sua maioria, com testes sorológicos, o que pode ocasionar falsos negativos e por consequência entre 20 a $30 \%$ dos indivíduos infectados desenvolverão como consequência grave as cardiomiopatias (RASSI Jr. et al 2015; MAYTA et al., 2019).

A dificuldade em diagnosticar a Doença de Chagas não ocorre quando se tem a fase aguda por transmissão oral, em razão da patologia geralmente ocorrer na forma de surtos (MENEZES et al., 2019). O crescimento das notificações desses surtos de Chagas aguda têm ocorrido pelas autoridades de saúde pública, que consideram a doença emergente na região Amazônia (BARBOSA et al., 2015) com possibilidade de expansão para outras regiões.

Para os surtos com notificação, a investigação epidemiológica diagnosticou transmissão não-vetorial, com associação a contaminação alimentar por frutas como Euterpe oleracea, conhecida popularmente como açaí e com amplo consumo como bebida na forma de sucos frescos (XAVIER et al., 2016). Na maioria das vezes pouco higienizada o que pode ocorrer durante a moagem do fruto com o vetor infectado, facilitando a transmissão da doeça e gerando surto da patologia (MENEZES et al., 2019). A transmissão por via oral do protozoário T. cruzi é 
considerada antiga, permitindo a circulação e manutenção do parasito no ciclo silvático ou enzoótico com ciclismo entre animais silvestres e vetores (HERNÁNDEZ et al., 2019). Prevalente principalmente entre mamíferos, a transmissão via oral, ocorre pela ingestão do vetor ou de dejetos deste contaminados com o protozoário (MENEZES et al., 2019). A patologia apresenta um período de incubação apresentado variação de acordo com a via de infecção, entretanto na fase aguda geralmente é observado de 3 a 21 dias (MEIS, 2017).

Dentre as principais manifestações clínicas, causadas pela Doença de Chagas na fase aguda, estão hipertermia constante e prolongada, mal-estar, cefaléia, astenia, linfonodos com aumento no volume discreto a moderado; hepatomegalia e/ou esplenomegalia, anemia, alterações eletrocardiográficas (BRASIL, 2018). O mecanismo de ação na transmissão oral ocorrer pela ação da proteína facilitadora gp82 que interage com a mucina gástrica, propiciando a invasão na mucosa gástrica do T.cruzi (STAQUICINI et al., 2010).

A fase aguda pela transmissão oral tem um relevante impacto na saúde pública em razão de ocorrer em surtos e poder levar pacientes a óbito. Programa de Saúde da Família, que tem como objetivo reorganização da atenção básica de acordo com os preceitos do Sistema Único de Saúde (SUS), tem que desenvolver estratégias de promoção, prevenção e controle, direcionada ao indivíduo, a família, ao trabalhador e a coletividade, por meio das políticas públicas de saúde, para reduzir a ingestão de alimentos contaminados (BRAGA NETO et al., 2014).

Desta forma se faz necessário uma busca ativa de estudos relacionados a transmissão da Doença de Chagas por alimentos contaminados. Os levantamentos de informações contribuem diretamente nos impactos da saúde pública, para o sistema de informação em saúde, além do desenvolvimento de tecnologias que possam contribuir com ações de estratégias de prevenção e de controle da doença.

Assim o trabalho teve por objetivo realizar uma revisão de literatura com intuito de identificar a incidência da transmissão via oral da Doença de Chagas por meio de alimentos contaminados e seus impactos no âmbito da saúde pública. 


\section{METODOLOGIA}

$\mathrm{Na}$ pesquisa por artigos científicos, buscou-se acesso online às bases de dados disponíveis e mais relevantes para o tema abordado, nos idiomas inglês, espanhol e português, como Science Direct, Portal de Periódicos da Coordenação de Aperfeiçoamento de Pessoal de Nível Superior (CAPES), Lilacs (Literatura Latino Americana em Ciências da Saúde), Scielo (Scientific Eletronic Library Online), Medline (National Library of Medicine and National Institutes of Health), PubMed, Biblioteca Virtual em Saúde (BVS) com os descritores “T. cruzi” "Epidemiologia” e/ou "Saúde Pública", no período de 19 de setembro de 2019 a 15 de fevereiro de 2020. Com a finalidade de fundamentr e ampliar os dados da pesquisa.

A escolha do material a ser utilizado na escrita, foi realizada de acordo com critérios como artigos gratuitos que estivessem disponíveis eletrônicamente na sua forma completa e mais recente, que tratassem de assuntos relacionados com o tema e com o objetivo do estudo, sendo eles referentes à Doença de Chagas, suas formas infectantes, dando ênfase na forma infectante por via oral.

Para exclusão foram adotador os seguintes criterios: Monografias, dissertções, teses, artigos que divergiam com a temática após leitura minussiosa dos seus resumos, assim como artigos que não disponibilizavam os seus resumos e estudos que se duplicavam nas bases de dados utilizadas para elaboração dessa pesquisa.

\section{RESULTADOS}

De acordo com o boletim epidemiológico descrito pelo Ministerio da Saúde, foi possível identificar, que os índices da Doença de Chagas têm aumentado de 129 casos (2012) para 298 casos ( 2016) por infecção via oral, o que representa $73 \%$ da forma de transmissão da Doença de Chagas aguda no período supracitado. 
(BRASIL, 2019). O que se configura um problema de saúde pública e faz com que essa transmissão seja a principal responsável pela patologia, inclusive acima da vetorial (MENEZES et al., 2019).

O aumento na transmissão por via oral foi observado em outros países da América Latina como Bolívia (VARGAS-CUENTAS et al., 2018), com relevante problema de saúde pública, estimado em 3milhões de pessoas acometidas pelo $T$. cruzi (2016) a secretaria de saúde pública boliviana, notificou pouco mais de 17 mil casos da doença, dos casos notificados $57 \%$ estão associados ao departamento de Santa cruz, que vem seguido por $23,13 \%$ dos pacientes infectados no periodo citado, encontram-se em Cochabamba (Vargas-Cuentas, 2018) . e na Colombia (RUEDA et al., 2014). Além várias regiões do Brasil (Tabela 1).

Sendo assim para que possa ter uma melhor visualização do panamorama atual sobre a tematica em analise, teve inicio o levantamento da amostragem como ferramente contribuir para uma melhor compressão do leitor no que se refere aos estudos analisados. A tabela 1 classifica os artigos de cordo com autor, titulo, períodico e objetivo de estudo. 
Tabela 1: Levantamento de dados das publicações da Doença de Chagas com transmissão via oral através de alimentos contaminados.

\begin{tabular}{|c|c|c|c|}
\hline $\begin{array}{c}\text { AUTORES/ANO } \\
\text { DE } \\
\text { PUBLICAÇÃO }\end{array}$ & TíTULO & PERIÓDICC & OBJETIVO \\
\hline $\begin{array}{l}\text { EERREIRA et } \\
\text { (2018) }\end{array}$ & $\begin{array}{l}\text { Detection and genotyping } \\
\text { al Trypanosoma cruzi from } \\
\text { açai products } \\
\text { commercialized in Rio de } \\
\text { Janeiro and Pará, Brazil }\end{array}$ & Scopus & $\begin{array}{l}\text { Investigação em açaí } \\
\text { comercializado no estado } \\
\text { do Rio de Janeiro e Pará }\end{array}$ \\
\hline $\begin{array}{l}=\text { EILIGHEDDUA } \epsilon \\
\text { al (2017) }\end{array}$ & $\begin{array}{l}\text { et Orally-transmitted Chagas } \\
\text { disease }\end{array}$ & $\begin{array}{l}\text { ScienceDire } \\
\text { ct }\end{array}$ & $\begin{array}{l}\text { Revisar a forma oral de } \\
\text { transmissão da doença de } \\
\text { chegas, e a importância do } \\
\text { desenvolvimento de } \\
\text { estratégias de prevenção e } \\
\text { controle da doença. }\end{array}$ \\
\hline $\begin{array}{l}\text { FRANCO- } \\
\text { PAREDES et } \\
(2020\end{array}$ & $\begin{array}{l}\text { A deadly feast: Elucidating } \\
\text { the burden of orally } \\
\text { al acquired acute Chagas } \\
\text { disease in Latin America - } \\
\text { Public health and travel } \\
\text { medicine importance }\end{array}$ & & $\begin{array}{l}\text { O estudo tem como } \\
\text { principal objetivo destacar } \\
\text { os aspectos } \\
\text { contemporâneos } \\
\text { doença da } \\
\text { adquirida por vira oral. }\end{array}$ \\
\hline $\begin{array}{l}\text { FUJITA et } \\
\text { (2019) }\end{array}$ & $\begin{array}{l}\text { The oral transmission of } \\
\text { al.chagas disease in Brazil: } \\
\text { New food supplies and } \\
\text { travel experience }\end{array}$ & & $\begin{array}{l}\text { Descrever o crescimento } \\
\text { da Doença de Chagas } \\
\text { transmitias por alimentos e } \\
\text { suas relações com viagens } \\
\text { turísticas. }\end{array}$ \\
\hline Gual & $\begin{array}{l}\text { Chagas disease as } \\
\text { 9) example of a reemerging } \\
\text { parasite }\end{array}$ & & $\begin{array}{l}\text { Busca apresentar um } \\
\text { panorama das mais } \\
\text { diversas vias de } \\
\text { transmissão da doença de } \\
\text { chagas, manifestações } \\
\text { clínicas, diagnósticos e } \\
\text { tratamentos. }\end{array}$ \\
\hline
\end{tabular}

\section{DISCUSSÕES}

O desenvolvimento da Doença de Chagas Aguda está relacionado com os hábitos de vida da população, há uma aproximação do homem com os fatores que condicionam para o surgimento da doença, como o crescimento das cidades, em 
áreas recentemente desmatadas, e também as condições socioeconômicas, como as condições ambientais nas quais os usuários do serviço de saúde estão expostos como, esgoto à céu aberto, acúmulo de lixo, ausência de saneamento básico (DEMEUS et al, 2019).

O estado do Rio Grande do Norte dentro de um cenário epidemiológico da doença em humanos pode ser considerado favorável no que se refere ao aumento populacional de triatomíneos encontrados, com ênfase a Triatoma brasiliensis associado algumas a outras espécies tem em processos de adaptação Triatoma pseudomaculata, encontrado em ambientes domiciliares o que demonstra uma maior adaptação o ao convívio humano (BARRETO et al, 2019).

Assim alguns alimentos têm apresentado uma maior vulnerabilidade de contaminação alimentar por T. cruzi : no Brasil destaque para o caldo da cana de açúcar e a poupa do açaí; na Colômbia contaminação em vinhos, palmitos e sucos de laranja; Venezuela em suco de goiaba; suco de "comou" presente na Guiana Francesa e o suco de majo na Bolívia (FILIGHEDDU et al, 2017).

Com isso FRANCO-PAREDES et al (2020) aponta ao concluir o seu estudo a necessidade dos agentes de turismo alertar os seus clientes com destino a regiões de risco, sobre as contaminação alimentar por t. cruzi, como forma de reduzir a vulnerabilidade de acometimento da doença de chagas humana por via oral. O autor ainda destaca a importância de alguns processos de produção de alimentos nas áreas citadas, como é o caso da pasteurização ou até mesmo realizar o procedimento de ferver a água na produção de bebidas.

Análises realizadas por FUJITA et al. (2019) demonstram que no Brasil o desenvolvimento da Doença de Chagas por via oral, tem representado um aumento de $60 \%$, onde a transmissão da doença por alimentos contaminados se caracterizam em forma de surtos, com os insetos responsáveis por transmitir a doença esmagados durante produção de alimentos ou as fezes dos vetores estão presentes na produção de insumos.

FUJITA e colaboradores (2019) ainda descreveram que a produção de açaí no Brasil durante o período de 2007 a 2016 teve um aumento considerável de $99,57 \%$, onde a Doença de Chagas por via oral acompanhou de maneira 
significativa esse crescimento desordenado no estado do Pará que apresentou no período de 2010: 13 casos e em 2014: 166 casos.

Com isso surge uma preocupação por meio das autoridades de que forma pode estar sendo produzido um alimento de qualidade buscando respeitar os aspectos sanitários, assim como a preocupação com os impactos na saúde pública e de que forma minimizar essa nova forma de transmissão da doença(FERREIRA et al., 2018).

BIVONNA e colaboradores (2020) consideram em seus estudos a importância da inclusão da vacinação na conduta terapêutica, pois se tornaria um recurso que não iria contribuir apenas para o surgimento de novos casos, mas que agregados a outras condutas terapêuticas, minimizaria as complicações relacionadas as cardiopatias, assim como a transmissão congênita.

Para tanto se faz necessário desenvolvimento de estratégias que fortaleça as ações de tratamento a pacientes infectados com $T$. cruzi, afim de minimizar os impactos na saúde pública, assim como estratégias de prevenção e controle em regiões não endêmicas de casos vetoriais, buscado disseminar o conhecimento acerca da forma de transmissão oral da doença, assim como viagens para regiões de risco e o desenvolvimento de medidas para diagnóstico precoce em caso de surtos ou epidemias (FILIGHEDDU et al, 2017).

O que também é descrito por ARAÚJO-NETO et al (2019), onde destaca a importância de um rastreamento de indivíduos contaminados pela doença, ainda ampliando a suas discussões para os reservatórios da doença como é o caso dos cães, destacando a necessidade de um conhecimento do perfil epidemiológico da em regiões endêmicas.

Se fazendo necessário a atuação da atenção primaria em saúde como porta de entrada ao serviço de saúde pública, no intuitivo de ofertar o tratamento aos pacientes infectados, além de contribuir para as ações de prevenção e controle, com isso também se faz necessário a participação do nível de assistência especializado buscando minimizar as complicações advindas da doença tais como: cardiopatias, alterações digestivas, pacientes submetidos a transplantes ou gestantes (BRASIL, 2018). 


\section{CONSIDERAÇÕES FINAIS}

Sendo assim é possível considerar que a transmissão por via oral vem ganhando um novo cenário, se fazendo necessário o desenvolvimento de políticas públicas que possam contribuir para o monitoramento da produção de alimentos, ofertando assim um alimento de qualidade conforme preconiza os aspectos sanitários, livre de risco de contaminação pelo Tt. cruzi, assim como ampliar o acesso ao diagnostico, tratamento, estratégias de prevenção e controle voltadas para a população, pois ainda sim é possível identificar indivíduos que desconhecem a doença, seus riscos e suas manifestações clínicas, tornando assim mais vulnerável a infecção.

Se torna essencial que o estudo permeei pelas mais diversas áreas de atuação, sendo ele de caráter interdisciplinar, tendo como direcionamento uma continuidade nas pesquisas cientificas, contribuindo de maneira significativa no avanço de recursos que possam estabelecer um controle da doença e que venha contribuir com uma melhor qualidade de vida aos pacientes já infectados.

\section{REFERÊNCIAS BIBLIOGRÁFICAS}

ARAUJO, P.F. et al., Sexual transmission of American trypanosomiasis in humans: a new potential pandemic route for Chagas parasites. Mem Inst Oswaldo Cruz. 2017 Jun;112(6):437446.

BARBOSA, M.G.V. et al. Chagas disease in the state of Amazonas: history, epidemiological evolution, risks of endemicity and future perspectives. Revista da Sociedade Brasileira de Medicina Tropical. v. 48, p. 27-33, 205.

BARRETO M. A. F.et al. Entomological triatomine indicators in the State of Rio Grande do Norte, Brazil. Ciencia \& Saude Coletiva, v.24, n. 4, p.1483-1493, 2019.

BIVONA, A. E. et al. Chagas disease vaccine design: the search for an efficient Trypanosoma cruzi immune-mediated control. Biochimica et Biophysica Acta (BBA) - Molecular Basis of Disease, p. 165658, 2020.

BRAGA NETO et al. Atenção hospitalar: evolução histórica e tendências. In: Políticas e sistema de saúde no Brasil. Rio de Janeiro: Fiocruz, p. 577-608, 2014.

BRASIL, Ministério da Saúde. Protocolo Clínico e diretrizes Terapêuticas Doença de 
Chagas. Secretaria de Ciência, Tecnologia e Insumos Estratégicos. 1 ed. Atual. - Brasília: Ministerio da Saúde, 145p., 2018.

BRASIL. Ministério da Saúde. DATAUS. Tecnologia de informação a serviço do sus. Notificação de casos de doença de chagas no Brasil. Disponível em <http://tabnet.datasus.gov.br/cgi/tabcgi.exe?sinannet/cnv/chagasbr.def>. acesso em: 19 de dezembro de 2019.

BRASIL. Ministério da Saúde. Secretaria de Vigilância em Saúde. Doença de Chagas Aguda e distribuição espacial dos triatomíneos de importância epidemiológica, Brasil 2012 a 2016. Boletim Epidemiológico, v. 50, n. 02, p. 1-10, 2018.

DE ARAÚJO-NETO, V. T. et al. Trypanosoma cruzi circulating among dogs and triatomines in the endemic countryside of the State of Rio Grande do Norte, Brazil. Acta tropica, v. 200, p. 105067, 2019.

DEMEU et al. Engineering a single-chain antibody against Trypanosoma cruzi metacyclic trypomastigotes to block cell invasion. Plos One. v.16, p. 1-15, 2019.

FERREIRA R. T. B. et al. Detection and genotyping of Trypanosoma cruzi from açai products commercialized in Rio de Janeiro and Pará, Brazil. Parasites \& vectors, v. 11, n. 1, p. 233, 2018.

FILIGHEDDU M. T. et al. Orally-transmitted Chagas disease. Medicina Clínica (English Edition), v. 148, n. 3, p. 125-131, 2017.

FRANCO-PAREDES, Carlos et al. A deadly feast: Elucidating the burden of orally acquired acute Chagas disease in Latin America-Public health and travel medicine importance. Travel Medicine and Infectious Disease, p. 101565, 2020.

FUJITAD. M. eet al. The oral transmission of chagas disease in brazil: new food supplies and travel experience. Acta tropica, 2019.

GÓMEZ, L. A. et al. Trypanosoma cruzi infection in transfusion medicine. Hematology, Transfusion and Cell Therapy. v. 41, n. 3, p:262-267, 2019.

HERNÁNDEZ C. et al. High-Resolution Molecular Typing of Trypanosoma cruzi in Large Outbreaks of Acute Chagas Disease in Colombia. The Journal of Infectious Diseases, v. 214, p. 1252-1255, 2016.

McCLEAN, M.C.W. et al. A lineage-specific rapid diagnostic test (Chagas Sero K-SeT) identifies Brazilian Trypanosoma cruzi II/V/VI reservoir hosts among diverse mammalian orders. PLoS One. v. 15, n.1, e0227828, 2020.

MEIS, J. Manual para diagnóstico em doenças de chagas para microscopista de base no estado do Para. Rio de Janeiro: FIOCRUZ, 110p., 2017.

MENEZES, L. R. et al., Epidemiological overview of chagas disease in the state of Amazonas, from 2004 to 2014. Revista de epidemiologia e controle de infecção. v. 9, n. 2, p. 116-121, 2019.

NEVES, D. P.; LINARDI, P. M.; VITOR, RWA. Parasitologia humana. $13^{a}$ edição. Rio de Janeiro: Atheneu, 2016.

RASSI JR, A. et al. American Trypanosomiasis (Chagas Disease). Infectious Disease Clinics of North America. v. 26, Issue 2, p. 275-291, 2012.

RIOS, L. et al. Epidemiology and pathogenesis of maternal-fetal transmission of Trypanosoma 
cruzi and a case for vaccine development against congenital Chagas disease. Biochimica et Biophysica Acta (BBA) - Molecular Basis of Disease. v. 1866, Issue 3, 165591, 2020.

RUEDA, K. et al. Transmisión oral de Trypanosoma cruzi: una nueva situación epidemiológica de la enfermedad de Chagas en Colombia y otros países suramericanos. Biomédica. v.34, p.631-41, 2014.

STAQUICINI, D. I. et al. Role of GP82 in the Selective Binding to Gastric Mucin during Oral Infection with Trypanosoma cruzi. PLoS Negl Trop Dis 4, e61. 2010.

VARGAS-CUENTAS, N. I. et al. Chagas disease study using satellite image processing: A Bolivian case. Acta Astronautica, v. 144, p. 216-224, 2018.

XAVIER, S.C.C. et al. Distantiae transmission of Trypanosoma cruzi: a new epidemiological feature of acute Chagas disease in Brazil. PLOS Neglected Tropical Diseases. v.8:e2878, 2014.

ZABALA, N.D.C et al. Trypanosoma cruzi infection in puerperal women and their neonates at Barcelona, Anzoategui State, Venezuela. Biomedica. v.39, n. 4, p.769-784, 2019. 